\title{
PITT'S INEQUALITY WITH SHARP CONVOLUTION ESTIMATES
}

\author{
WILLIAM BECKNER
}

(Communicated by Michael T. Lacey)

\begin{abstract}
Sharp $L^{p}$ extensions of Pitt's inequality expressed as a weighted Sobolev inequality are obtained using convolution estimates and Stein-Weiss potentials. Optimal constants are obtained for the full Stein-Weiss potential as a map from $L^{p}$ to itself which in turn yield semi-classical Rellich inequalities on $\mathbb{R}^{n}$. Additional results are obtained for Stein-Weiss potentials with gradient estimates and with mixed homogeneity. New proofs are given for the classical Pitt and Stein-Weiss estimates.
\end{abstract}

Weighted inequalities for the Fourier transform provide a natural measure to characterize both uncertainty and the balance between functional growth and smoothness. On $\mathbb{R}^{n}$ the question is to determine sharp quantitative comparisons between the relative size of a function and its Fourier transform at infinity. Pitt's inequality illustrates this principle at the spectral level (see [4], [7]):

$$
\int_{\mathbb{R}^{n}} \Phi(1 /|x|)|f(x)|^{2} d x \leq C_{\Phi} \int_{\mathbb{R}^{n}} \Phi(|y|)|\hat{f}(y)|^{2} d y
$$

where $\Phi$ is an increasing function, $f$ is in the Schwartz class $\mathcal{S}\left(\mathbb{R}^{n}\right)$ and the Fourier transform is defined by

$$
(\mathcal{F} f)(y)=\hat{f}(y)=\int_{\mathbb{R}^{n}} e^{2 \pi i x y} f(x) d x .
$$

The objective here is to extend Pitt's inequality to $L^{p}\left(\mathbb{R}^{n}\right)$ for $1<p<\infty$ in the form

$$
\int_{\mathbb{R}^{n}}|\Phi(1 /|x|) f(x)|^{p} d x \leq C_{\Phi, p} \int_{\mathbb{R}^{n}}|\Phi(\sqrt{-\Delta}) f(x)|^{p} d x
$$

where $\Delta$ denotes the Laplacian on $\mathbb{R}^{n}$. Sharp constants for Pitt's inequality can be calculated using Stein-Weiss potentials and Young's inequality as in the equivalent results $([4])$ :

Pitt's inequality. For $f \in \mathcal{S}\left(\mathbb{R}^{n}\right)$ and $0 \leq \alpha<n$

$$
\begin{gathered}
\int_{\mathbb{R}^{n}}|x|^{-\alpha}|f(x)|^{2} d x \leq C_{\alpha} \int_{\mathbb{R}^{n}}|y|^{\alpha}|\hat{f}(y)|^{2} d y \\
C_{\alpha}=\pi^{\alpha}\left[\Gamma\left(\frac{n-\alpha}{4}\right) / \Gamma\left(\frac{n+\alpha}{4}\right)\right]^{2} .
\end{gathered}
$$

Received by the editors December 19, 2006.

2000 Mathematics Subject Classification. Primary 58J70, 42B10, 35A15. 
Stein-Weiss potentials. For $f \in L^{2}\left(\mathbb{R}^{n}\right)$ and $0<\alpha<n$

$$
\begin{gathered}
\left|\int_{\mathbb{R}^{n} \times \mathbb{R}^{n}} f(x) \frac{1}{|x|^{\alpha / 2}} \frac{1}{|x-y|^{n-\alpha}} \frac{1}{|y|^{\alpha / 2}} f(y) d x d y\right| \leq B_{\alpha} \int_{\mathbb{R}^{n}}|f(x)|^{2} d x, \\
B_{\alpha}=\pi^{n / 2}\left[\Gamma\left(\frac{\alpha}{2}\right) / \Gamma\left(\frac{n-\alpha}{2}\right)\right]\left[\Gamma\left(\frac{n-\alpha}{4}\right) / \Gamma\left(\frac{n+\alpha}{4}\right)\right]^{2} .
\end{gathered}
$$

Alternate proofs of inequality (3) are given in [11] and [24]. This Stein-Weiss potential corresponds to a linear operator in $L^{2}\left(\mathbb{R}^{n}\right)$

$$
g \rightarrow|x|^{-\alpha / 2}\left(|x|^{-(n-\alpha / 2)} * g\right)
$$

and inequality (4) can be rephrased as

$$
\left\||x|^{-\alpha / 2}\left(|x|^{-(n-\alpha / 2)} * g\right)\right\|_{L^{2}\left(\mathbb{R}^{n}\right)} \leq \pi^{n / 2}\left[\frac{\Gamma\left(\frac{\alpha}{4}\right) \Gamma\left(\frac{n-\alpha}{4}\right)}{\Gamma\left(\frac{2 n-\alpha}{4}\right) \Gamma\left(\frac{n+\alpha}{4}\right)}\right]\|g\|_{L^{2}\left(\mathbb{R}^{n}\right)},
$$

where $0<\alpha<n$. It is this inequality that extends naturally to $L^{p}\left(\mathbb{R}^{n}\right)$ using the Hardy-Littlewood paradigm that a positive integral operator that commutes with dilations may be reduced to Young's inequality for sharp convolution estimates on the multiplicative group (see discussion on bilinear forms and integrals in [14], chapter 9). It is important to recognize that obtaining optimal norm constants for $L^{p}$ inequalities is inherent in the approach. This method has been applied by the author in several articles, including [4], [5] and [7], but it was used earlier by Herbst in his study of the Klein-Gordon equation for a Coulomb potential (see Theorem 2.5 and the related discussion in [15]).

Theorem 1 (Herbst). For $g \in L^{p}\left(\mathbb{R}^{n}\right)$ and $h \in L^{p^{\prime}}\left(\mathbb{R}^{n}\right)$ with $1<p<\infty, 1 / p+$ $1 / p^{\prime}=1$ and $0<\alpha<n$

$$
\begin{gathered}
\left\||x|^{-\alpha / p}\left(|x|^{-(n-\alpha / p)} * g\right)\right\|_{L^{p}\left(\mathbb{R}^{n}\right)} \leq C_{\alpha, p}\|g\|_{L^{p}\left(\mathbb{R}^{n}\right)}, \\
\left\||x|^{-(n-\alpha / p)} *\left(|x|^{-\alpha / p} h\right)\right\|_{L^{p^{\prime}\left(\mathbb{R}^{n}\right)}} \leq C_{\alpha, p}\|h\|_{L^{p^{\prime}\left(\mathbb{R}^{n}\right)}}, \\
C_{\alpha, p}=\pi^{n / 2}\left[\frac{\Gamma\left(\frac{\alpha}{2 p}\right) \Gamma\left(\frac{n-\alpha}{2 p}\right) \Gamma\left(\frac{n}{2 p^{\prime}}\right)}{\Gamma\left(\frac{n}{2}-\frac{\alpha}{2 p}\right) \Gamma\left(\frac{n}{2 p^{\prime}}+\frac{\alpha}{2 p}\right) \Gamma\left(\frac{n}{2 p}\right)}\right] .
\end{gathered}
$$

Proof. Observe that (7) follows from (6) by duality. By using rearrangement and symmetrization, both inequalities are reduced to non-negative radial decreasing functions. Set $t=|x|, k(t)=|x|^{n / p} g(|x|), \sigma=\frac{n}{p}-\frac{n}{2}-\frac{\alpha}{2 p}$ and

$$
\psi_{\sigma}(t)=t^{\sigma} \int_{S^{n-1}}\left(t+\frac{1}{t}-2 \xi_{1}\right)^{-\left(\sigma+n / p^{\prime}\right)} d \omega
$$

where $d \omega$ is surface measure on $S^{n-1}$ and $\xi_{1}$ is the first component of $\xi \in S^{n-1}$. Note that $\sigma$ can take both positive and negative values, but $\sigma+\frac{n}{p^{\prime}}=\frac{n}{2}-\frac{\alpha}{2 p}>0$ and $\psi_{\sigma} \in L^{r}\left(\mathbb{R}_{+}\right)$for $r \geq 1$. Then inequality (6) corresponds to

$$
\left\|\psi_{\sigma} * k\right\|_{L^{p}\left(\mathbb{R}_{+}\right)} \leq\left\|\psi_{\sigma}\right\|_{L^{1}\left(\mathbb{R}_{+}\right)}\|k\|_{L^{p}\left(\mathbb{R}_{+}\right)}
$$


with $C_{\alpha, p}=\left\|\psi_{\sigma}\right\|_{L^{1}\left(\mathbb{R}_{+}\right)}$.

$$
\begin{aligned}
\left\|\psi_{\sigma}\right\|_{L^{1}\left(\mathbb{R}_{+}\right)} & =\int_{0}^{\infty} \psi_{\sigma}(t) \frac{1}{t} d t=\int_{\mathbb{R}^{n}}|x-\xi|^{-(n-\alpha / p)}|x|^{-\left(n / p^{\prime}+\alpha / p\right)} d x \\
& =\pi^{n / 2}\left[\frac{\Gamma\left(\frac{\alpha}{2 p}\right) \Gamma\left(\frac{n-\alpha}{2 p}\right) \Gamma\left(\frac{n}{2 p^{\prime}}\right)}{\Gamma\left(\frac{n}{2}-\frac{\alpha}{2 p}\right) \Gamma\left(\frac{n}{2 p^{\prime}}+\frac{\alpha}{2 p}\right) \Gamma\left(\frac{n}{2 p}\right)}\right],
\end{aligned}
$$

where $|\xi|=1$.

As outlined in the introduction, this result can be expressed as a weighted Sobolev inequality. Because such estimates have the same dilation character as Hardy's inequality, they have been classified as Hardy-Rellich inequalities, and in addition they can be viewed in the framework of the Maz'ya-Eilertsen inequality [11]. The following corollary to Theorem 1 generalizes equation (20) in [7]. The techniques developed here allow the extension of the results from [7] on iterated Stein-Weiss potentials from $L^{2}\left(\mathbb{R}^{n}\right)$ to $L^{p}\left(\mathbb{R}^{n}\right)$ (see also Corollary 14 in [10]).

Corollary 1. For $g \in \mathcal{S}\left(\mathbb{R}^{n}\right), 1<p<\infty, 0 \leq \alpha<n$,

$$
\left\||x|^{-\alpha / p} g\right\|_{L^{p}\left(\mathbb{R}^{n}\right)} \leq 2^{-\alpha / p}\left[\frac{\Gamma\left(\frac{n-\alpha}{2 p}\right) \Gamma\left(\frac{n}{2 p^{\prime}}\right)}{\Gamma\left(\frac{n}{2 p^{\prime}}+\frac{\alpha}{2 p}\right) \Gamma\left(\frac{n}{2 p}\right)}\right]\left\|(-\Delta)^{\alpha / 2 p} g\right\|_{L^{p}\left(\mathbb{R}^{n}\right)} .
$$

This result recovers the classical Hardy-Rellich inequality for $1<p<n / 2$ :

$$
\int_{\mathbb{R}^{n}}|x|^{-2 p}|g|^{p} d x \leq\left[\frac{p p^{\prime}}{n(n-2 p)}\right]^{p} \int_{\mathbb{R}^{n}}|\Delta g|^{p} d x .
$$

Corollary 2 (Logarithmic uncertainty). For $g \in \mathcal{S}\left(\mathbb{R}^{n}\right), 1<p<\infty$,

$$
\begin{array}{r}
\int_{\mathbb{R}^{n}} \ln |x||g|^{p} d x+\int_{\mathbb{R}^{n}}(\ln \sqrt{-\Delta}) g g|g|^{p-2} d x \geq D \int_{\mathbb{R}^{n}}|g|^{p} d x, \\
D=\ln 2+\frac{1}{2}\left[\psi\left(\frac{n}{2 p}\right)+\psi\left(\frac{n}{2 p^{\prime}}\right)\right],
\end{array}
$$

where $\psi=(\ln \Gamma)^{\prime}$.

This result follows directly from equation (9) by a differentiation argument since the constant is identically one with equality at $\alpha=0$. Moreover, it extends the uncertainty principle derived in [4].

The full Stein-Weiss inequality has a more general character which includes varying powers of weights (see Appendix). Both [17] and [23] suggest that the diagonal map on $L^{p}\left(\mathbb{R}^{n}\right)$ has more difficult aspects, but in fact the inherent convolution structure ensures an immediate sharp reduction to radial functions, and the lack of extremal functions facilitates the calculation of optimal constants.

Theorem 2. For $f \in L^{p}\left(\mathbb{R}^{n}\right)$ with $1<p<\infty, 0<\lambda<n, \alpha<n / p, \beta<n / p^{\prime}$ and $n=\lambda+\alpha+\beta$,

$$
\begin{gathered}
\left\||x|^{-\alpha}\left(|x|^{-\lambda} *\left(|x|^{-\beta} f\right)\right)\right\|_{L^{p}\left(\mathbb{R}^{n}\right)} \leq D_{\alpha, \beta}\|f\|_{L^{p}\left(\mathbb{R}^{n}\right)}, \\
D_{\alpha, \beta}=\pi^{n / 2}\left[\frac{\Gamma\left(\frac{\alpha+\beta}{2}\right) \Gamma\left(\frac{n}{2 p}-\frac{\alpha}{2}\right) \Gamma\left(\frac{n}{2 p^{\prime}}-\frac{\beta}{2}\right)}{\Gamma\left(\frac{n-\alpha-\beta}{2}\right) \Gamma\left(\frac{n}{2 p^{\prime}}+\frac{\alpha}{2}\right) \Gamma\left(\frac{n}{2 p}+\frac{\beta}{2}\right)}\right] .
\end{gathered}
$$


Proof. Note that it is not required that both $\alpha$ and $\beta$ are non-negative, but rather $0<\alpha+\beta<n$. By setting $t=|x|$ and $\sigma=\frac{n}{p}-\alpha-\frac{\lambda}{2}$, inequality (11) has an equivalent formulation as a convolution estimate on the product manifold $\mathbb{R}_{+} \times$ $S^{n-1}$ :

$$
\left\|K_{\sigma, \lambda} * h\right\|_{L^{p}\left(\mathbb{R}_{+} \times S^{n-1}\right)} \leq\left\|K_{\sigma, \lambda}\right\|_{L^{1}\left(\mathbb{R}_{+} \times S^{n-1}\right)}\|h\|_{L^{p}\left(\mathbb{R}_{+} \times S^{n-1}\right)},
$$

where $h(t, \xi)=|x|^{n / p} f(|x|, \xi)$ and $K_{\sigma, \lambda}(t, \xi \cdot \eta)=t^{\sigma}\left(t+\frac{1}{t}-2 \xi \cdot \eta\right)^{-\lambda / 2}$ with $\xi, \eta \in S^{n-1}$. Then

$$
\begin{aligned}
D_{\alpha, \beta} & =\left\|K_{\sigma, \lambda}\right\|_{L^{1}\left(\mathbb{R}_{+}, S^{n-1}\right)}=\int_{\mathbb{R}_{+} \times S^{n-1}} t^{\sigma+\lambda / 2}\left(t^{2}+1-2 t \xi_{1}\right)^{-\lambda / 2} d \omega \frac{d t}{t} \\
& =\int_{\mathbb{R}^{n}}|x-\xi|^{-\lambda}|x|^{-\left(\alpha+n / p^{\prime}\right)} d x \\
& =\pi^{n / 2}\left[\frac{\Gamma\left(\frac{\alpha+\beta}{2}\right) \Gamma\left(\frac{n}{2 p}-\frac{\alpha}{2}\right) \Gamma\left(\frac{n}{2 p^{\prime}}-\frac{\beta}{2}\right)}{\Gamma\left(\frac{n-\alpha-\beta}{2}\right) \Gamma\left(\frac{n}{2 p^{\prime}}+\frac{\alpha}{2}\right) \Gamma\left(\frac{n}{2 p}+\frac{\beta}{2}\right)}\right],
\end{aligned}
$$

where $\xi$ is a unit vector with $\xi_{1}$ its first component and $d \omega$ denotes surface measure on $S^{n-1}$.

Considerable interest has been given recently to the study of Hardy, Rellich and weighted Sobolev inequalities on complete Riemannian manifolds with optimal constants and remainder terms (for example, see [1], [10], [13]). Davies and Hinz proved the following two $L^{p}$ inequalities on $\mathbb{R}^{n}$ (see Theorems 12 and 13 in [10]) which can be extended using the results and methods for the Stein-Weiss inequality from Theorem 2 above:

$$
\begin{gathered}
\int_{\mathbb{R}^{n}}|x|^{-\gamma}\left|\Delta^{m} u\right|^{p} d x \geq A_{\gamma, m} \int_{\mathbb{R}^{n}}|x|^{-\gamma-2 m p}|u|^{p} d x \\
\int_{\mathbb{R}^{n}}|x|^{-\gamma}\left|\nabla \Delta^{m} u\right|^{p} d x \geq B_{\gamma, m} \int_{\mathbb{R}^{n}}|x|^{-\gamma-(2 m+1) p}|u|^{p} d x
\end{gathered}
$$

for suitable restrictions on $\gamma$ and $m$.

Theorem 3. For $g \in \mathcal{S}\left(\mathbb{R}^{n}\right)$ with $1<p<\infty, 0<\alpha+\beta<n, \alpha<n / p$, and $\beta<n / p^{\prime}$,

$$
\left\||x|^{-\alpha} g\right\|_{L^{p}\left(\mathbb{R}^{n}\right)} \leq 2^{-(\alpha+\beta)}\left[\frac{\Gamma\left(\frac{n}{2 p}-\frac{\alpha}{2}\right) \Gamma\left(\frac{n}{2 p^{\prime}}-\frac{\beta}{2}\right)}{\Gamma\left(\frac{n}{2 p^{\prime}}+\frac{\alpha}{2}\right) \Gamma\left(\frac{n}{2 p}+\frac{\beta}{2}\right)}\right]\left\||x|^{\beta}(-\Delta)^{(\alpha+\beta) / 2} g\right\|_{L^{p}\left(\mathbb{R}^{n}\right)} .
$$

Proof. This result follows directly from Theorem 2. To obtain equation (12), set $\beta=-\gamma / p, \alpha=\gamma / p+2 m$. Then $\alpha+\beta=2 m$ and

$$
1 / A_{\gamma, m}=2^{-2 m p}\left[\frac{\Gamma\left(\frac{n-\gamma}{2 p}-m\right) \Gamma\left(\frac{n}{2 p^{\prime}}+\frac{\gamma}{2 p}\right)}{\Gamma\left(\frac{n}{2 p^{\prime}}+\frac{\gamma}{2 p}+m\right) \Gamma\left(\frac{n-\gamma}{2 p}\right)}\right]^{p} .
$$


Theorem 4 (Stein-Weiss potentials with gradient estimates). For $f, g \in \mathcal{S}\left(\mathbb{R}^{n}\right)$, $1<p<\infty, 0<\lambda<n, \alpha<n / p, 1-n / p<\beta<n / p^{\prime}$ and $n+1=\lambda+\alpha+\beta+\sigma$,

$$
\begin{gathered}
\left\||x|^{-\alpha}\left(|x|^{-\lambda} *\left(|x|^{-\sigma} f\right)\right)\right\|_{L^{p}\left(\mathbb{R}^{n}\right)} \leq F_{\alpha, \beta, \sigma}\left\||x|^{\beta} \nabla f\right\|_{L^{p}\left(\mathbb{R}^{n}\right)}, \\
F_{\alpha, \beta, \sigma}=\pi^{n / 2}\left(\frac{n}{p}+\beta-1\right)^{-1}\left[\frac{\Gamma\left(\frac{\alpha+\beta+\sigma-1}{2}\right) \Gamma\left(\frac{n}{2 p}-\frac{\alpha}{2}\right) \Gamma\left(\frac{n}{2 p^{\prime}}-\frac{\beta+\sigma-1}{2}\right)}{\Gamma\left(\frac{n+1-\alpha-\beta-\sigma}{2}\right) \Gamma\left(\frac{n}{2 p^{\prime}}+\frac{\alpha}{2}\right) \Gamma\left(\frac{n}{2 p}+\frac{\beta+\sigma-1}{2}\right)}\right], \\
\left\||x|^{-\alpha} g\right\|_{L^{p}\left(\mathbb{R}^{n}\right)} \leq \frac{2^{-(\alpha+\beta-1)}}{n / p+\beta-1}\left[\frac{\Gamma\left(\frac{n}{2 p}-\frac{\alpha}{2}\right) \Gamma\left(\frac{n}{2 p^{\prime}}-\frac{\beta+\sigma-1}{2}\right)}{\Gamma\left(\frac{n}{2 p^{\prime}}+\frac{\alpha}{2}\right) \Gamma\left(\frac{n}{2 p}+\frac{\beta+\sigma-1}{2}\right)}\right] \\
\cdot\left\||x|^{\beta} \nabla\left(|x|^{\sigma}(-\Delta)^{(\alpha+\beta-1) / 2} g\right)\right\|_{L^{p}\left(\mathbb{R}^{n}\right)} .
\end{gathered}
$$

Proof. Note that $1<\alpha+\beta+\sigma<n+1$. The argument can be reduced to radial functions by setting

$$
h(|x|)=\left[\int_{S^{n-1}}|f(x)|^{p} d \omega\right]^{1 / p},|\nabla h| \leq\left[\int_{S^{n-1}}|\nabla f|^{p} d \omega\right]^{1 / p},
$$

and then applying Young's inequality on $S^{n-1}$ to obtain

$$
\begin{gathered}
\left\||x|^{-\alpha}\left(|x|^{-\lambda} *\left(|x|^{-\sigma} f\right)\right)\right\|_{L^{p}\left(\mathbb{R}^{n}\right)} \leq\left\||x|^{-\alpha}\left(|x|^{-\lambda} *\left(|x|^{-\sigma} h\right)\right)\right\|_{L^{p}\left(\mathbb{R}^{n}\right)} \\
\leq F_{\alpha, \beta, \sigma}\left\||x|^{\beta} \nabla h\right\|_{L^{p}\left(\mathbb{R}^{n}\right)} \leq F_{\alpha, \beta, \sigma}\left\||x|^{\beta} \nabla f\right\|_{L^{p}\left(\mathbb{R}^{n}\right)^{.}}
\end{gathered}
$$

To obtain the inner inequality, set $t=|x|, u=|x|^{\beta+n / p}\left|h^{\prime}\right|$ and observe that the resulting inequality is now a convolution inequality on the multiplicative group $\mathbb{R}_{+}$

$$
\begin{aligned}
& \left\|K_{1} * K_{2} * u\right\|_{L^{p}\left(\mathbb{R}_{+}\right)} \leq\left\|K_{1} * K_{2}\right\|_{L^{1}\left(\mathbb{R}_{+}\right)}\|u\|_{L^{p}\left(\mathbb{R}_{+}\right)}, \\
& F_{\alpha, \beta, \sigma}=\left\|K_{1} * K_{2}\right\|_{L^{1}\left(\mathbb{R}_{+}\right)}=\left\|K_{1}\right\|_{L^{1}\left(\mathbb{R}_{+}\right)}\left\|K_{2}\right\|_{L^{1}\left(\mathbb{R}_{+}\right)}
\end{aligned}
$$

with

$$
\begin{gathered}
K_{1}(t)=t^{n / p-\alpha-\lambda / 2} \int_{S^{n-1}}\left(t+\frac{1}{t}-2 \xi_{1}\right)^{-\lambda / 2} d \omega, \\
K_{2}(t)=t^{n / p+\beta-1} \chi_{[0.1]}(t), \quad\left\|K_{2}\right\|_{L^{1}\left(\mathbb{R}_{+}\right)}=\left(\frac{n}{p}+\beta-1\right)^{-1}, \\
\left\|K_{1}\right\|_{L^{1}\left(\mathbb{R}_{+}\right)}=\int_{0}^{\infty} K_{1}(t) \frac{1}{t} d t=\int_{\mathbb{R}^{n}}|x-\xi|^{-\lambda}|x|^{-\left(\alpha+n / p^{\prime}\right)} d x \\
=\pi^{n / 2}\left[\frac{\Gamma\left(\frac{\alpha+\beta+\sigma-1}{2}\right) \Gamma\left(\frac{n}{2 p}-\frac{\alpha}{2}\right) \Gamma\left(\frac{n}{2 p^{\prime}}-\frac{\beta+\sigma-1}{2}\right)}{\Gamma\left(\frac{n+1-\alpha-\beta-\sigma}{2}\right) \Gamma\left(\frac{n}{2 p^{\prime}}+\frac{\alpha}{2}\right) \Gamma\left(\frac{n}{2 p}+\frac{\beta+\sigma-1}{2}\right)}\right]
\end{gathered}
$$

so that

$$
F_{\alpha, \beta, \sigma}=\pi^{n / 2}\left(\frac{n}{p}+\beta-1\right)^{-1}\left[\frac{\Gamma\left(\frac{\alpha+\beta+\sigma-1}{2}\right) \Gamma\left(\frac{n}{2 p}-\frac{\alpha}{2}\right) \Gamma\left(\frac{n}{2 p^{\prime}}-\frac{\beta+\sigma-1}{2}\right)}{\Gamma\left(\frac{n+1-\alpha-\beta-\sigma}{2}\right) \Gamma\left(\frac{n}{2 p^{\prime}}+\frac{\alpha}{2}\right) \Gamma\left(\frac{n}{2 p}+\frac{\beta+\sigma-1}{2}\right)}\right] .
$$

To obtain equation (13), set $\alpha=\gamma / p+2 m+1, \beta=-\gamma / p$ and $\sigma=0$. Then $\alpha+\beta-1=2 m$ and

$$
1 / B_{\gamma, m}=2^{-(2 m+1) p}\left[\frac{\Gamma\left(\frac{n-\gamma}{2 p}-m-\frac{1}{2}\right) \Gamma\left(\frac{n}{2 p^{\prime}}+\frac{\gamma}{2 p}+\frac{1}{2}\right)}{\Gamma\left(\frac{n}{2 p^{\prime}}+\frac{\gamma}{2 p}+m+\frac{1}{2}\right) \Gamma\left(\frac{n-\gamma}{2 p}+\frac{1}{2}\right)}\right]^{p} .
$$


Problems with mixed homogeneity have been of interest in relation to embedding estimates for differential operators. Motivated by earlier work (see [5], [6], [8]) centered on Grushin symmetry and Carnot geometry, and the more recent work by Barbatis [1], corresponding estimates are obtained for Stein-Weiss potentials on Euclidean product manifolds with differing homogeneity. Because of the concentration phenomenon that occurs for the sharp $L^{1}$ Young's inequality, one can apply either Euclidean analysis or the underlying hyperbolic symmetry to obtain optimal estimates.

Theorem 5 (Stein-Weiss potentials with mixed homogeneity). For $f \in L^{p}\left(\mathbb{R}^{n+m}\right)$, $1<p<\infty, w=(x, v) \in \mathbb{R}^{n} \times \mathbb{R}^{m}, 0<\lambda<n+m, \alpha<m / p, \beta<m / p^{\prime}$ and $n+m=\lambda+\alpha+\beta$,

$$
\begin{gathered}
\left\||v|^{-\alpha}\left(|w|^{-\lambda} *\left(|v|^{-\beta} f\right)\right)\right\|_{L^{p}\left(\mathbb{R}^{n+m}\right)} \leq G_{\alpha, \beta}\|f\|_{L^{p}\left(\mathbb{R}^{n+m}\right)}, \\
G_{\alpha, \beta}=\pi^{(n+m) / 2}\left[\frac{\Gamma\left(\frac{\alpha+\beta}{2}\right) \Gamma\left(\frac{m}{2 p}-\frac{\alpha}{2}\right) \Gamma\left(\frac{m}{2 p^{\prime}}-\frac{\beta}{2}\right)}{\Gamma\left(\frac{n+m-\alpha-\beta}{2}\right) \Gamma\left(\frac{m}{2 p^{\prime}}+\frac{\alpha}{2}\right) \Gamma\left(\frac{m}{2 p}+\frac{\beta}{2}\right)}\right] .
\end{gathered}
$$

Proof. Apply the sharp $L^{1}$ Young's inequality for convolution on $\mathbb{R}^{n}$ followed by the Stein-Weiss argument from Theorem 2 for the $\mathbb{R}^{m}$ variable. That is,

$$
\begin{aligned}
& \left\||v|^{-\alpha}\left(|w|^{-\lambda} *\left(|v|^{-\beta} f\right)\right)\right\|_{L^{p}\left(\mathbb{R}^{n+m}\right)} \leq\left\||v|^{-\alpha}\left(J *\left(|v|^{-\beta} u\right)\right)\right\|_{L^{p}\left(\mathbb{R}^{m}\right)} \\
& =\pi^{n / 2}\left[\frac{\Gamma\left(\frac{(m-\alpha-\beta)}{2}\right)}{\Gamma\left(\frac{n+m-\alpha-\beta}{2}\right)}\right]\left\||v|^{-\alpha}\left(|v|^{-\lambda^{\prime}} *\left(|v|^{-\beta} u\right)\right)\right\|_{L^{p}\left(\mathbb{R}^{m}\right)} \\
& \leq \pi^{(n+m) / 2}\left[\frac{\Gamma\left(\frac{\alpha+\beta}{2}\right) \Gamma\left(\frac{m}{2 p}-\frac{\alpha}{2}\right) \Gamma\left(\frac{m}{2 p^{\prime}}-\frac{\beta}{2}\right)}{\Gamma\left(\frac{n+m-\alpha-\beta}{2}\right) \Gamma\left(\frac{m}{2 p^{\prime}}+\frac{\alpha}{2}\right) \Gamma\left(\frac{m}{2 p}+\frac{\beta}{2}\right)}\right]\|u\|_{L^{p}\left(\mathbb{R}^{m}\right)} \\
& =\pi^{(n+m) / 2}\left[\frac{\Gamma\left(\frac{\alpha+\beta}{2}\right) \Gamma\left(\frac{m}{2 p}-\frac{\alpha}{2}\right) \Gamma\left(\frac{m}{2 p^{\prime}}-\frac{\beta}{2}\right)}{\Gamma\left(\frac{n+m-\alpha-\beta}{2}\right) \Gamma\left(\frac{m}{2 p^{\prime}}+\frac{\alpha}{2}\right) \Gamma\left(\frac{m}{2 p}+\frac{\beta}{2}\right)}\right]\|f\|_{L^{p}\left(\mathbb{R}^{n+m}\right)},
\end{aligned}
$$

where $\lambda^{\prime}=\lambda-n=m-\alpha-\beta$ and

$$
u(v)=\left[\int_{\mathbb{R}^{n}}|f(x, v)|^{p} d x\right]^{1 / p}, \quad\|u\|_{L^{p}\left(\mathbb{R}^{m}\right)}=\|f\|_{L^{p}\left(\mathbb{R}^{n+m}\right)}
$$

with

$$
J(v)=\int_{\mathbb{R}^{n}}\left(x^{2}+v^{2}\right)^{-\lambda / 2} d x=|v|^{-(\lambda-n)} \frac{\pi^{n / 2}}{\Gamma(n / 2)} \int_{0}^{\infty} t^{n / 2-1}(1+t)^{-\lambda / 2} d t .
$$

An alternate proof can be given by recognizing that the inequality reduces to radial functions in the $v$-variable and rewriting the inequality to be on $\mathbb{R}^{n+1}$ with $y=$ $|v|>0$, and then transferring the resulting inequality to the $(n+1)$-dimensional hyperbolic space $\mathbb{H}^{n+1}$ with left-invariant Haar measure $d \nu=y^{-n-1} d x d y$. The result follows by applying the sharp $L^{1}$ Young's inequality for convolution on a non-unimodular group. This curious aspect of multiplicity of proofs simply reflects the complexity of the symmetry (see [5], [8]). 
Corollary 1. For $g \in \mathcal{S}\left(\mathbb{R}^{n+m}\right)$ with $w=(x, v) \in \mathbb{R}^{n} \times \mathbb{R}^{m}, 1<p<\infty, 0<$ $\alpha+\beta<m, \alpha<m / p$, and $\beta<m / p^{\prime}$,

$$
\begin{aligned}
&\left\||v|^{-\alpha} g\right\|_{L^{p}\left(\mathbb{R}^{n+m}\right)} \leq 2^{-(\alpha+\beta)}\left[\frac{\Gamma\left(\frac{m}{2 p}-\frac{\alpha}{2}\right) \Gamma\left(\frac{m}{2 p^{\prime}}-\frac{\beta}{2}\right)}{\Gamma\left(\frac{m}{2 p^{\prime}}+\frac{\alpha}{2}\right) \Gamma\left(\frac{m}{2 p}+\frac{\beta}{2}\right)}\right] \\
& \cdot\left\||v|^{\beta}(-\Delta)^{(\alpha+\beta) / 2} g\right\|_{L^{p}\left(\mathbb{R}^{n+m}\right)}
\end{aligned}
$$

Similar arguments to those used for Theorem 5 give gradient estimates with mixed homogeneity:

Corollary 2. For $g \in \mathcal{S}\left(\mathbb{R}^{n+m}\right)$ with $w=(x, v) \in \mathbb{R}^{n} \times \mathbb{R}^{m}, 1<p<\infty$, $0<$ $\alpha+\beta<m, \alpha<m / p$, and $\beta<m / p^{\prime}$,

$$
\begin{aligned}
&\left\||v|^{-\alpha} g\right\|_{L^{p}\left(\mathbb{R}^{n+m}\right)} \leq 2^{-(\alpha+\beta)}\left[\frac{\Gamma\left(\frac{m}{2 p}-\frac{\alpha}{2}\right) \Gamma\left(\frac{m}{2 p^{\prime}}-\frac{\beta}{2}+\frac{1}{2}\right)}{\Gamma\left(\frac{m}{2 p^{\prime}}+\frac{\alpha}{2}\right) \Gamma\left(\frac{m}{2 p}+\frac{\beta}{2}+\frac{1}{2}\right)}\right] \\
& \cdot\left\||v|^{\beta} \nabla(-\Delta)^{(\alpha+\beta-1) / 2} g\right\|_{L^{p}\left(\mathbb{R}^{n+m}\right)}
\end{aligned}
$$

Riesz potentials can be viewed as extending Young's inequality to include weak Lebesgue classes. Stein-Weiss potentials extend Riesz potentials by incorporating successive multiplication by fractional powers - first on the function side and then followed on the Fourier transform side with repetitions. Such iterations include both extensions of Young's inequality and the framework of the Maz'ya-Eilertsen inequality [11]. The following theorem extends from $L^{2}\left(\mathbb{R}^{n}\right)$ to $L^{p}\left(\mathbb{R}^{n}\right)$ the results on iterated Stein-Weiss potentials from [7].

Theorem 6. For $f \in L^{p}\left(\mathbb{R}^{n}\right), 1<p<\infty, \alpha+\sigma=\beta+\rho, 0<\alpha<n / p^{\prime}, 0<\sigma<n$, $-n / p<\rho-\sigma$, and $\rho<n / p^{\prime}$,

$$
\begin{gathered}
\left\||x|^{-(n-\alpha)} *\left[|x|^{-\beta}\left(|x|^{-(n-\sigma)} *\left(|x|^{-\rho} f\right)\right)\right]\right\|_{L^{p}\left(\mathbb{R}^{n}\right)} \leq E_{\alpha, \sigma, \rho}\|f\|_{L^{p}\left(\mathbb{R}^{n}\right)}, \\
E_{\alpha, \sigma, \rho}=\pi^{n}\left[\frac{\Gamma\left(\frac{\alpha}{2}\right) \Gamma\left(\frac{\sigma}{2}\right) \Gamma\left(\frac{n}{2 p}\right) \Gamma\left(\frac{n}{2 p^{\prime}}-\frac{\alpha}{2}\right) \Gamma\left(\frac{n}{2 p^{\prime}}-\frac{\rho}{2}\right) \Gamma\left(\frac{n}{2 p}+\frac{\rho}{2}-\frac{\sigma}{2}\right)}{\Gamma\left(\frac{n-\alpha}{2}\right) \Gamma\left(\frac{n-\sigma}{2}\right) \Gamma\left(\frac{n}{2 p^{\prime}}\right) \Gamma\left(\frac{n}{2 p}+\frac{\alpha}{2}\right) \Gamma\left(\frac{n}{2 p}+\frac{\rho}{2}\right) \Gamma\left(\frac{n}{2 p^{\prime}}-\frac{\rho}{2}+\frac{\sigma}{2}\right)}\right] .
\end{gathered}
$$

Proof. Set $\lambda=\frac{n}{2}-\frac{n}{p^{\prime}}+\rho-\frac{\sigma}{2}$ and $\nu=\frac{n}{p}-\frac{n}{2}+\frac{\alpha}{2}$. Then inequality (20) has an equivalent formulation as a convolution estimate on the product manifold $\mathbb{R}_{+} \times$ $S^{n-1}$ :

$$
\begin{gathered}
\left\|K_{1} * K_{2} * h\right\|_{L^{p}\left(\mathbb{R}_{+} \times S^{n-1}\right)} \leq\left\|K_{1} * K_{2}\right\|_{L^{1}\left(\mathbb{R}_{+} \times S^{n-1}\right)}\|h\|_{L^{p}\left(\mathbb{R}_{+} \times S^{n-1}\right)}, \\
E_{\alpha, \sigma, \rho}=\left\|K_{1} * K_{2}\right\|_{L^{1}\left(\mathbb{R}_{+} \times S^{n-1}\right)}=\left\|K_{1}\right\|_{L^{1}\left(\mathbb{R}_{+} \times S^{n-1}\right)}\left\|K_{2}\right\|_{L^{1}\left(\mathbb{R}_{+} \times S^{n-1}\right)},
\end{gathered}
$$


where $h(t, \xi)=|x|^{n / p} f(|x|, \xi)$ with $t=|x|$ and

$$
\begin{aligned}
K_{1}(t, \xi \cdot \eta) & =t^{\nu}\left(t+\frac{1}{t}-2 \xi \cdot \eta\right)^{-(n-\alpha) / 2}, \\
K_{2}(t, \xi \cdot \eta) & =t^{\lambda}\left(t+\frac{1}{t}-2 \xi \cdot \eta\right)^{-(n-\sigma) / 2}, \\
\left\|K_{1}\right\|_{L^{1}\left(\mathbb{R}_{+} \times S^{n-1}\right)} & =\int_{\mathbb{R}_{+} \times S^{n-1}} t^{\nu+(n-\alpha) / 2}\left(t^{2}+1-2 t \xi_{1}\right)^{-(n-\alpha) / 2} d \omega \frac{d t}{t} \\
& =\int_{\mathbb{R}^{n}}|x-\xi|^{-(n-\alpha)}|x|^{-(n+\alpha) / 2+\nu} d x \\
& =\int_{\mathbb{R}^{n}}|x-\xi|^{-(n-\alpha)}|x|^{-n / p^{\prime}} d x \\
& =\pi^{n / 2}\left[\frac{\Gamma\left(\frac{\alpha}{2}\right) \Gamma\left(\frac{n}{2 p}\right) \Gamma\left(\frac{n}{2 p^{\prime}}-\frac{\alpha}{2}\right)}{\Gamma\left(\frac{n-\alpha}{2}\right) \Gamma\left(\frac{n}{2 p^{\prime}}\right) \Gamma\left(\frac{n}{2 p}+\frac{\alpha}{2}\right)}\right] \\
\left\|K_{2}\right\|_{L^{1}\left(\mathbb{R}_{+} \times S^{n-1}\right)} & =\int_{\mathbb{R}_{+} \times S^{n-1}} t^{\lambda+(n-\sigma) / 2}\left(t^{2}+1-2 t \xi_{1}\right)^{-(n-\sigma) / 2} d \omega \frac{d t}{t} \\
& =\int_{\mathbb{R}^{n}}|x-\xi|^{-(n-\sigma)}|x|^{-(n+\sigma) / 2+\lambda} d x \\
& =\int_{\mathbb{R}^{n}}|x-\xi|^{-(n-\sigma)}|x|^{-n / p^{\prime}+\rho-\sigma} d x \\
& \pi^{n / 2}\left[\frac{\Gamma\left(\frac{\sigma}{2}\right) \Gamma\left(\frac{n}{2 p}+\frac{\rho}{2}-\frac{\sigma}{2}\right) \Gamma\left(\frac{n}{2 p^{\prime}}-\frac{\rho}{2}\right)}{\Gamma\left(\frac{n-\sigma}{2}\right) \Gamma\left(\frac{n}{2 p^{\prime}}+\frac{\sigma}{2}-\frac{\rho}{2}\right) \Gamma\left(\frac{n}{2 p}+\frac{\rho}{2}\right)}\right] \cdot
\end{aligned}
$$

Then

$$
E_{\alpha, \sigma, \rho}=\pi^{n}\left[\frac{\Gamma\left(\frac{\alpha}{2}\right) \Gamma\left(\frac{\sigma}{2}\right) \Gamma\left(\frac{n}{2 p}\right) \Gamma\left(\frac{n}{2 p^{\prime}}-\frac{\alpha}{2}\right) \Gamma\left(\frac{n}{2 p^{\prime}}-\frac{\rho}{2}\right) \Gamma\left(\frac{n}{2 p}+\frac{\rho}{2}-\frac{\sigma}{2}\right)}{\Gamma\left(\frac{n-\alpha}{2}\right) \Gamma\left(\frac{n-\sigma}{2}\right) \Gamma\left(\frac{n}{2 p^{\prime}}\right) \Gamma\left(\frac{n}{2 p}+\frac{\alpha}{2}\right) \Gamma\left(\frac{n}{2 p}+\frac{\rho}{2}\right) \Gamma\left(\frac{n}{2 p^{\prime}}-\frac{\rho}{2}+\frac{\sigma}{2}\right)}\right] .
$$

Note that as in the original Stein-Weiss inequality, $\beta$ and $\rho$ can take negative values as long as $\beta+\rho$ is positive. Two lemmas are used in the above calculation of the optimal constant. The first lemma is obtained using the inverse Fourier transform for fractional powers.

Lemma 1. For $0<\lambda<n, 0<\mu<n$ and $\lambda+\mu>n$,

$$
|x|^{-\lambda} *|x|^{-\mu}=\pi^{n / 2}\left[\frac{\Gamma\left(\frac{n-\lambda}{2}\right) \Gamma\left(\frac{n-\mu}{2}\right) \Gamma\left(\frac{\lambda+\mu-n}{2}\right)}{\Gamma\left(\frac{\lambda}{2}\right) \Gamma\left(\frac{\mu}{2}\right) \Gamma\left(\frac{2 n-\lambda-\mu}{2}\right)}\right]|x|^{-(\lambda+\mu-n)} .
$$

Lemma 2. For $F \in L^{p}\left(S^{n}\right), G \in L^{p^{\prime}}\left(S^{n}\right)$ and $K(\xi \cdot \eta) \geq 0$ with $\xi, \eta \in S^{n}$,

$$
\begin{gathered}
\left|\int_{S^{n} \times S^{n}} G(\xi) K(\xi \cdot \eta) F(\eta) d \xi d \eta\right| \leq\left(\int_{S^{n}} K\left(\xi_{1}\right) d \xi\right)\|F\|_{L^{p}\left(S^{n}\right)}\|G\|_{L^{p^{\prime}\left(S^{n}\right)}}, \\
\|K * F\|_{L^{p}\left(S^{n}\right)} \leq\|K\|_{L^{1}\left(S^{n}\right)}\|F\|_{L^{p}\left(S^{n}\right)} .
\end{gathered}
$$

Proof. Split the integrand in (22) into the product of two terms, $F K^{1 / p}$ and $G K^{1 / p^{\prime}}$, and apply Hölder's inequality on the product manifold $S^{n} \times S^{n}$. 
Corollary 1 (Iterated Hardy-Rellich inequality). For $h \in \mathcal{S}\left(\mathbb{R}^{n}\right), 1<p<n$, and the parameters $\alpha, \beta, \sigma, \rho$ as specified in Theorem 6 ,

$$
\begin{gathered}
\|h\|_{L^{p}\left(\mathbb{R}^{n}\right)} \leq C\left\||x|^{\rho}(-\Delta)^{\sigma / 2}|x|^{\beta}(-\Delta)^{\alpha / 2} h\right\|_{L^{p}\left(\mathbb{R}^{n}\right)}, \\
C=2^{-(\alpha+\sigma)}\left[\frac{\Gamma\left(\frac{n}{2 p}\right) \Gamma\left(\frac{n}{2 p^{\prime}}-\frac{\alpha}{2}\right) \Gamma\left(\frac{n}{2 p^{\prime}}-\frac{\rho}{2}\right) \Gamma\left(\frac{n}{2 p}+\frac{\rho}{2}-\frac{\sigma}{2}\right)}{\Gamma\left(\frac{n}{2 p^{\prime}}\right) \Gamma\left(\frac{n}{2 p}+\frac{\alpha}{2}\right) \Gamma\left(\frac{n}{2 p}+\frac{\rho}{2}\right) \Gamma\left(\frac{n}{2 p^{\prime}}-\frac{\rho}{2}+\frac{\sigma}{2}\right)}\right] .
\end{gathered}
$$

As with Riesz potentials, iterated Stein-Weiss potentials coupled with symmetrization provide extensions of Young's inequality to include weak Lebesgue classes:

$$
h \in L_{q, \infty}\left(\mathbb{R}^{n}\right) \Longleftrightarrow h^{*}(x) \leq \frac{A}{|x|^{n / q}}, \quad 0<q<\infty,
$$

where $h^{*}$ denotes the equimeasurable radial decreasing rearrangement of $|h|$ on $\mathbb{R}^{n}$.

Corollary 2 (Young's inequality). For $f \in L^{p}\left(\mathbb{R}^{n}\right), 1<p<\infty, 1<q_{k}<\infty$, $\sum 1 / q_{k}=2, q_{1}<p, q_{4}>p^{\prime}$,

$$
\left\|h_{1} * h_{2}\left(h_{3} *\left(h_{4} f\right)\right)\right\|_{L^{p}\left(\mathbb{R}^{n}\right)} \leq C \prod_{k=1}^{4}\left\|h_{k}\right\|_{L_{q_{k, \infty}}\left(\mathbb{R}^{n}\right)}\|f\|_{L^{p}\left(\mathbb{R}^{n}\right)} .
$$

By using the methods outlined in the Appendix (section 3), one obtains results from the Stein-Weiss theorem for Young's inequality that include off-diagonal $L^{p}-$ $L^{q}$ maps:

Theorem 7. For $f \in L^{p}\left(\mathbb{R}^{n}\right), 1<p \leq q<\infty, 1<r_{k}<\infty, 1 / q+1 / p^{\prime}=\sum 1 / r_{k}$, $r_{1}>q, 1<q_{k}<\infty, 1+1 / q+1 / p^{\prime}=\sum 1 / q_{k}, q_{1}<q, q_{4}>p^{\prime}$,

$$
\begin{gathered}
\left\|g_{1}\left(g_{2} *\left(g_{3} f\right)\right)\right\|_{L^{q}\left(\mathbb{R}^{n}\right)} \leq C \prod_{k=1}^{3}\left\|g_{k}\right\|_{L_{r_{k, \infty}}\left(\mathbb{R}^{n}\right)}\|f\|_{L^{p}\left(\mathbb{R}^{n}\right)}, \\
\left\|h_{1} * h_{2}\left(h_{3} *\left(h_{4} f\right)\right)\right\|_{L^{q}\left(\mathbb{R}^{n}\right)} \leq C \prod_{k=1}^{4}\left\|h_{k}\right\|_{L_{q_{k, \infty}}\left(\mathbb{R}^{n}\right)}\|f\|_{L p\left(\mathbb{R}^{n}\right)} .
\end{gathered}
$$

This theorem easily generalizes to include multiple applications of linear operators defined by multiplication followed by convolution of functions in weak Lebesgue classes, e.g.,

$$
L_{k} f=g_{2 k} *\left(g_{2 k-1} f\right), T f=\left(\prod_{k} L_{k}\right) f .
$$

Remark. The constants obtained in Theorems 1, 2, 3, 4, 5 and 6 are optimal and no extremal functions exist for the corresponding variational problems. This circumstance follows directly from recognition that dilation invariance implies an equivalent formulation as sharp convolution estimates on $\mathbb{R}_{+} \times S^{n-1}$ with a positive integrable kernel. After this paper was finished, the author learned that Theorems 2 and 3 had been obtained independently by Samko [19].

\section{APPENDIX}

Three arguments are developed here that contribute to a broader setting for the results in this note. A short proof of the classical Pitt's inequality on $\mathbb{R}^{n}$ for $n \geq 2$ is given by applying rearrangement inequalities, convolution estimates and 
interpolation. Pitt's inequality can be viewed as a generalized Hausdorff-Young inequality with weights. From that context, it is not surprising that the only cases where optimal constants have been calculated correspond to the Hausdorff-Young inequality, the Hardy-Littlewood-Sobolev inequality, and the spectral-level diagonal maps defined by Stein-Weiss potentials.

The second issue addressed here is to show the connection between an argument taken from Stein and Weiss [23] and the Hardy-Littlewood paradigm relating dilation invariance for a positive linear operator to sharp convolution estimates on the multiplicative group. To fully complete the background for these problems, a short proof of the Stein-Weiss theorem on fractional integrals is given using Young's inequality. The structural correspondence between Pitt's inequality and the SteinWeiss theorem underlines the essential duality between the Fourier transform and convolution. More broadly, the essential idea is that identification of global symmetry facilitates not simply calculation of the mathematical estimates but also a fuller understanding of the analytic framework.

\section{Pitt's Inequality}

Pitt's inequality. For $f \in \mathcal{S}\left(\mathbb{R}^{n}\right), 1<p \leq q<\infty, 0<\alpha<n / q, 0<\beta<n / p^{\prime}$ and $n \geq 2$,

$$
\left[\left.\left.\int_{\mathbb{R}^{n}}|| x\right|^{-\alpha} \hat{f}\right|^{q} d x\right]^{1 / q} \leq A\left[\left.\left.\int_{\mathbb{R}^{n}}|| x\right|^{\beta} f\right|^{p} d x\right]^{1 / p}
$$

with the index constraint

$$
\frac{n}{p}+\frac{n}{q}+\beta-\alpha=n
$$

(note that primes denote dual exponents, $1 / p+1 / p^{\prime}=1$ ).

Proof. The argument will be accomplished in stages.

Step 1. Observe that by duality, inequality (28) for $p<q$ implies the dual inequality

$$
\left[\left.\left.\int_{\mathbb{R}^{n}}|| x\right|^{-\beta} \hat{h}\right|^{p^{\prime}} d x\right]^{1 / p^{\prime}} \leq A\left[\left.\left.\int_{\mathbb{R}^{n}}|| x\right|^{\alpha} h\right|^{q^{\prime}} d x\right]^{1 / q^{\prime}},
$$

which simply reverses the place of $\alpha$ and $\beta$ with $q^{\prime}<p^{\prime}$.

Step 2. As a preliminary calculation, consider (28) for $q=p^{\prime}$, which requires $\beta-\alpha=0$. Then using the Hausdorff-Young inequality combined with Theorem 1 ,

$$
\begin{aligned}
{\left[\left.\left.\int_{\mathbb{R}^{n}}|| x\right|^{-\alpha} \hat{f}\right|^{q} d x\right]^{1 / q} } & \leq C_{1}\left[\left.\left.\int_{\mathbb{R}^{n}}|| x\right|^{-(n-\alpha)} * f\right|^{p} d x\right]^{1 / p} \\
& \leq C_{2}\left[\left.\left.\int_{\mathbb{R}^{n}}|| x\right|^{\alpha} f\right|^{p} d x\right]^{1 / p}
\end{aligned}
$$

which demonstrates (28) for the case $q=p^{\prime}$ and $0<\alpha<n / q$.

Step 3. For $q=2$ and $\beta=0$, then (28) corresponds to the Hardy-LittlewoodSobolev inequality. But it is surprising to note that the case of positive $\beta$ does not follow from this result. For $\beta>0$ and $q=2$, Pitt's inequality is equivalent to

$$
\left[\left.\left.\int_{\mathbb{R}^{n}}|| x\right|^{-(n-\alpha)} *\left(|x|^{-\beta} f\right)\right|^{2} d x\right]^{1 / 2} \leq B\left[\int_{\mathbb{R}^{n}}|f|^{p} d x\right]^{1 / p}
$$


with $\frac{n}{2}-\alpha=\frac{n}{p^{\prime}}-\beta$. Using symmetrization and rearrangement, this estimate is reduced to non-negative radial decreasing functions on $\mathbb{R}^{n}$. Set $t=|x|$ and $h(t)=|x|^{-n / p} f(x)$. Now the above inequality is equivalent to

$$
\left\|\psi_{\alpha} * h\right\|_{L^{2}\left(\mathbb{R}_{+}\right)} \leq C\|h\|_{L^{p}\left(\mathbb{R}_{+}\right)}
$$

where

$$
\psi_{\alpha}(t)=t^{\alpha / 2} \int_{S^{n-1}}\left(t+t^{-1}-2 \xi_{1}\right)^{-(n-\alpha) / 2} d \xi
$$

with $d \xi$ being normalized surface measure on $S^{n-1}, \xi_{1}$ denoting the first component of $\xi$ and Haar measure on $\mathbb{R}_{+}$being $t^{-1} d t$. Since $\psi_{\alpha} \in L^{r}\left(\mathbb{R}_{+}\right)$for all $r \geq 1$, inequality (28) for this case follows from Young's inequality

$$
\left\|\psi_{\alpha} * h\right\|_{L^{2}\left(\mathbb{R}_{+}\right)} \leq\left\|\psi_{\alpha}\right\|_{L^{r}\left(\mathbb{R}_{+}\right)}\|h\|_{L^{p}\left(\mathbb{R}_{+}\right)}
$$

for $1 / 2=1 / p+1 / r-1$.

Step 4 . The central step in the proof is to show that (28) holds whenever $q$ is an even integer, $q=2 m$ with $m \geq 2$. Then

$$
\begin{gathered}
\left.\left.\int_{\mathbb{R}^{n}}|| x\right|^{-\alpha} \hat{f}\right|^{2 m} d x=c \int_{\mathbb{R}^{n}}\left|\left(|x|^{-(n-\alpha)} * f\right) * \cdots *\left(|x|^{-(n-\alpha)} * f\right)\right|^{2} d x \\
\leq c \int_{\mathbb{R}^{n}}\left|\left(|x|^{-(n-\alpha)} * f^{*}\right) * \cdots *\left(|x|^{-(n-\alpha)} * f^{*}\right)\right|^{2} d x
\end{gathered}
$$

where $f^{*}$ denotes the radial equimeasurable decreasing rearrangement of $|f|$, and there are $m$ convolutions in each string appearing in the integral above, and for any $p>0$ and $\alpha>0$

$$
\left.\left.\int_{\mathbb{R}^{n}}|| x\right|^{\alpha} f^{*}\right|^{p} d x \leq\left.\left.\int_{\mathbb{R}^{n}}|| x\right|^{\alpha} f\right|^{p} d x
$$

since $|x|^{\alpha}$ is an increasing function. Hence for $q=2 m$, it suffices to consider (28) for non-negative radial decreasing functions. The most direct approach at this point is to apply Young's inequality successively — first on $\mathbb{R}^{n}$ and then on $\mathbb{R}_{+}$. Inequality (28) is equivalent to

$$
\left[\left.\left.\int_{\mathbb{R}^{n}}|| x\right|^{-\alpha}\left(|x|^{-\beta} f\right)^{\wedge}\right|^{2 m} d x\right]^{1 / 2 m} \leq A\left[\int_{\mathbb{R}^{n}}|f|^{p} d x\right]^{1 / p}
$$

and

$$
\begin{aligned}
& {\left[\left.\left.\int_{\mathbb{R}^{n}}|| x\right|^{-\alpha}\left(|x|^{-\beta} f\right)^{\wedge}\right|^{2 m} d x\right]^{1 / 2 m}} \\
& \quad=c\left[\int_{\mathbb{R}^{n}}\left|\left(|x|^{-(n-\alpha)} *|x|^{-\beta} f\right) * \cdots *\left(|x|^{-(n-\alpha)} *|x|^{-\beta} f\right)\right|^{2} d x\right]^{1 / 2 m} \\
& \quad \leq c\left\||x|^{-(n-\alpha)} *|x|^{-\beta} f\right\|_{L^{r}\left(\mathbb{R}^{n}\right)}
\end{aligned}
$$

by applying Young's inequality on $\mathbb{R}^{n}$ for $r=2 m /(2 m-1)$. The proof for this case is finished by showing that

$$
\left\||x|^{-(n-\alpha)} *|x|^{-\beta} f\right\|_{L^{r}\left(\mathbb{R}^{n}\right)} \leq C_{1}\|f\|_{L^{p}\left(\mathbb{R}^{n}\right)}
$$

with $n / r=(\beta-\alpha)+n / p$. It suffices to show this inequality for radial functions $f$, and that case is controlled by Young's inequality on $\mathbb{R}_{+}$

$$
\left\|\psi_{\sigma, \alpha} * h\right\|_{L^{r}\left(\mathbb{R}_{+}\right)} \leq\left\|\psi_{\sigma,, \alpha}\right\|_{L^{s}\left(\mathbb{R}_{+}\right)}\|h\|_{L^{p}\left(\mathbb{R}_{+}\right)},
$$


where $\frac{1}{r}=\frac{1}{s}+\frac{1}{p}-1, r=2 m /(2 m-1), \sigma=\frac{n}{2}-\frac{n}{2 m}+\frac{\alpha}{2}$ and

$$
\psi_{\sigma, \alpha}=t^{\sigma} \int_{S^{n-1}}\left(t+t^{-1}-2 \xi_{1}\right)^{-(n-\alpha) / 2} d \xi
$$

with $d \xi$ being a normalized surface measure on $S^{n-1}$ and $\xi_{1}$ the first component of $\xi$. $\psi_{\sigma, \alpha} \in L^{s}\left(\mathbb{R}_{+}\right)$for any $s \geq 1$ if $(n-\alpha) / 2-\sigma=n / 2 m-\alpha=n / q-\alpha>0$, which is a necessary restriction for Pitt's inequality. Another approach for $m \geq 2$ would be to use the fact that the Fourier transform of a radial function results in a transform with a kernel having some decay at infinity:

$$
\int_{\mathbb{R}^{n}} e^{2 \pi i x y} f(|y|) d y=c \int_{0}^{\infty}(|x||y|)^{-(n / 2-1)} J_{n / 2-1}(2 \pi|x||y|) f(|y|) d y .
$$

Here $J_{n / 2-1}$ is a Bessel function of the first kind. Then inequality (28) for radial functions

$$
\left\||x|^{-\alpha}\left(|x|^{-\beta} f\right)^{\wedge}\right\|_{L^{q}\left(\mathbb{R}^{n}\right)} \leq A\|f\|_{L^{p}\left(\mathbb{R}^{n}\right)}
$$

corresponds to the convolution inequality on $\mathbb{R}_{+}$

$$
\|K * h\|_{L^{q}\left(\mathbb{R}_{+}\right)} \leq B\|h\|_{L^{p}\left(\mathbb{R}_{+}\right)}
$$

with $K(t)=t^{\sigma+1-n / 2} J_{n / 2-1}(t) \sigma=\frac{n}{q}-\alpha=\frac{n}{p^{\prime}}-\beta>0$. If $q$ is at least 4 , then $K \in L^{r}\left(\mathbb{R}_{+}\right)$for all $r \geq 1$. By Young's inequality, one can take $B=\|K\|_{L^{r}\left(\mathbb{R}_{+}\right)}$ with $\frac{1}{r}=\frac{1}{q}+\frac{1}{p^{\prime}}$.

Step 5. The proof of Pitt's inequality is completed by applying Riesz-Stein complex interpolation to show that the linear operator

$$
\left(T_{\alpha, \beta} f\right)(x)=|x|^{-\alpha}\left(|x|^{-\beta} f\right)^{\wedge}(x)
$$

is bounded from $L^{p}\left(\mathbb{R}^{n}\right)$ to $L^{q}\left(\mathbb{R}^{n}\right)$ on the convex domain $\frac{n}{q}-\alpha=\frac{n}{p^{\prime}}-\beta, q \geq p$, $q \geq 2,0<\alpha<\frac{n}{q}$, and $0<\beta<\frac{n}{p^{\prime}}$. The operator $T_{\alpha, \beta}$ is an analytic function of both $\alpha$ and $\beta$. Every point in the above set lies on a line connecting two values of $q$ that are even integers. Using Steps 3 and 4, which give the required endpoint bounds, Pitt's inequality holds for all parameters in the above 3-dimensional convex domain (see lemma on page 385 in [22]). Duality from Step 1 extends the result to values $p<q<2$, and the proof of $(28)$ is finished.

\section{Stein-Weiss LEMma}

The Hardy-Littlewood paradigm relating dilation invariance for a positive integral operator to sharp convolution estimates on the multiplicative group is used to clarify an old argument by Stein and Weiss (see Lemma 2.1 in [23]).

Stein-Weiss lemma. Suppose $K$ is a non-negative kernel defined on $\mathbb{R}^{n} \times \mathbb{R}^{n}$, continuous on any domain that excludes the point $(0,0)$, homogeneous of degree $-n, K(\delta u, \delta v)=\delta^{-n} K(u, v)$, and $K(R u, R v)=K(u, v)$ for any $R \in S O(n)$. Then $K$ defines an integral operator

$$
(T f)(x)=\int_{\mathbb{R}^{n}} K(x, y) f(y) d y
$$

which maps $L^{p}\left(\mathbb{R}^{n}\right)$ to $L^{p}\left(\mathbb{R}^{n}\right)$ for $1<p<\infty$

$$
\|T f\|_{L^{p}\left(\mathbb{R}^{n}\right)} \leq A\|f\|_{L^{p}\left(\mathbb{R}^{n}\right)},
$$


where the optimal constant is given by

$$
A=\int_{\mathbb{R}^{n}} K\left(x, \hat{e}_{1}\right)|x|^{-n / p^{\prime}} d x,
$$

and $\hat{e}_{1}$ is a unit vector in the first coordinate direction.

Proof. Set $s=|y|, t=|x|, h(s)=s^{n / p}\left[\int_{S^{n-1}}|f(s \xi)|^{p} d \xi\right]^{1 / p}$ and $\bar{K}(t, s)=$ $\int_{S^{n-1}} K\left(t \xi, s \hat{e}_{1}\right) d \sigma$. Then using the invariance of $K$ with respect to the rotation group

$$
\begin{aligned}
\|T f\|_{L^{p}\left(\mathbb{R}^{n}\right)} & \leq\left[\int_{0}^{\infty}\left|t^{n / p} \int_{0}^{\infty} \bar{K}(t, s) s^{n / p^{\prime}} h(s) \frac{d s}{s}\right|^{p} \frac{d t}{t}\right]^{1 / p} \\
& =\left[\int_{0}^{\infty}\left|\int_{0}^{\infty}\left(\frac{t}{s}\right)^{n / p} \bar{K}\left(\frac{t}{s}, 1\right) h(s) \frac{d s}{s}\right|^{p} \frac{d t}{t}\right]^{1 / p} \\
& =\|\psi * h\|_{L^{p}\left(\mathbb{R}_{+}\right)} \leq\|\psi\|_{L^{1}\left(\mathbb{R}_{+}\right)}\|h\|_{L^{p}\left(\mathbb{R}_{+}\right)},
\end{aligned}
$$

where $\psi(t)=t^{n / p} \bar{K}(t, 1),\|h\|_{L^{p}\left(\mathbb{R}_{+}\right)}=\|f\|_{L^{p}\left(\mathbb{R}^{n}\right)}$ and

$$
\|\psi\|_{L^{1}\left(\mathbb{R}_{+}\right)}=\int_{\mathbb{R}^{n}} K\left(x, \hat{e}_{1}\right)|x|^{-n / p^{\prime}} d x
$$

one can see that $\|\psi\|_{L^{1}\left(\mathbb{R}_{+}\right)}$is the optimal constant by considering radial functions.

\section{Stein-Weiss THEOREM}

Stein-Weiss theorem. For $f \in \mathcal{S}\left(\mathbb{R}^{n}\right), 1<p \leq q<\infty, 0<\lambda<n, \alpha<n / q$, $\beta<n / p^{\prime}, \alpha+\beta \geq 0$ and $\frac{n}{q}+\frac{n}{p^{\prime}}=\lambda+\alpha+\beta$,

$$
\left\||x|^{-\alpha}\left(|x|^{-\lambda} * f\right)\right\|_{L^{q}\left(\mathbb{R}^{n}\right)} \leq A\left\||x|^{\beta} f\right\|_{L^{p}\left(\mathbb{R}^{n}\right)} .
$$

Proof. Observe that because $\alpha$ and $\beta$ are not both necessarily non-negative but only the sum is non-negative, there is not a reduction to radial functions. But it is still possible to express this problem as a product convolution on $\mathbb{R}_{+} \times S^{n-1}$ where convolution on the symmetric space $S^{n-1}$ is defined by

$$
(K * F)(\xi)=\int_{S^{n-1}} K(\xi \cdot n) F(\eta) d \eta
$$

and $K$ is defined on the interval $(-1,1)$. The standard convolution estimates expressed by W.H. Young's inequality hold in this symmetric space setting (see Zygmund [25]: Section 1.15, ch. 2, vol. I). Then inequality (31) is determined by

$$
\left\|K_{\sigma} * h\right\|_{L^{q}\left(\mathbb{R}_{+} \times S^{n-1}\right)} \leq\left\|K_{\sigma}\right\|_{L^{r}\left(\mathbb{R}_{+} \times S^{n-1}\right)}\|h\|_{L^{p}\left(\mathbb{R}_{+} \times S^{n-1}\right)},
$$

where $h(t, \xi)=|x|^{\beta+n / p} f(|x|, \xi)$ with $t=|x|, \sigma=\frac{n}{q}-\alpha-\frac{\lambda}{2}=-\left(\frac{n}{p^{\prime}}-\beta-\frac{\lambda}{2}\right)$, and $1 / r=1 / q+1 / p^{\prime}=(\lambda+\alpha+\beta) / n$. To obtain the Stein-Weiss theorem, one need only check that

$$
K_{\sigma}(t, \xi \cdot \eta)=t^{\sigma}\left(t+\frac{1}{t}-2 \xi \cdot \eta\right)^{-\lambda / 2} \in L^{r}\left(\mathbb{R}_{+} \times S^{n-1}\right),
$$

where $r=n /(\lambda+\alpha+\beta)$. Note that $r \lambda<n$. Calculate

$$
\int_{\mathbb{R}_{+} \times S^{n-1}}\left|K_{\sigma}\left(t, \xi_{1}\right)\right|^{r} d \omega \frac{d t}{t}=\int_{\mathbb{R}^{n}} \frac{1}{|x-\eta|^{r \lambda}} \frac{1}{|x|^{n-r(n / q-\alpha)}} d x<\infty
$$


if $0<\lambda<n$ and $-n / q^{\prime}<\alpha<n / q$ which follows from $-n / q^{\prime}<-n / p^{\prime}<-\beta<$ $\alpha<n / q$, and this completes the proof of the Stein-Weiss theorem.

\section{ACKNOWLEDGMENTS}

I would like to thank Michael Perelmuter for drawing my attention to the work of Herbst [15] and the paper [16] where the spectral properties of Schrödinger operators acting on $L^{p}$ spaces are studied. Emanuel Carneiro was especially helpful in reviewing all of the arguments used here.

\section{REFERENCES}

[1] G. Barbatis, Best constants for higher-order Rellich inequalities in $L^{p}(\Omega)$, Math. Z., 255 (2007) 877-896. MR2274540

[2] W. Beckner, Inequalities in Fourier analysis, Annals Math. 102 (1975), 159-182. MR0385456 (52:6317)

[3] W. Beckner, Geometric inequalities in Fourier analysis, Essays on Fourier Analysis in Honor of Elias M. Stein, Princeton University Press, 1995, pp. 36-68. MR1315541 (95m:42004)

[4] W. Beckner, Pitt's inequality and the uncertainty principle, Proc. Amer. Math. Soc. 123 (1995), 1897-1905. MR1254832 (95g:42021)

[5] W. Beckner, Sharp inequalities and geometric manifolds, J. Fourier Anal. Appl. 3 (1997), 825-836. MR1600195 (2000c:58059)

[6] W. Beckner, On the Grushin operator and hyperbolic symmetry, Proc. Amer. Math. Soc. 129 (2001), 1233-1246. MR1709740 (2001g:35009)

[7] W. Beckner, Weighted inequalities and Stein-Weiss potentials, Forum Math. (in press).

[8] W. Beckner, Grushin symmetry - embedding and potentials (preprint, 2007).

[9] H. J. Brascamp, E. H. Lieb and J. M. Luttinger, A general rearrangement inequality for multiple integrals, J. Funct. Anal. 17 (1974), 227-237. MR0346109 (49:10835)

[10] E. B. Davies and A. M. Hinz, Explicit constants for Rellich inequalities in $L_{p}(\Omega)$, Math. Z. 227 (1998), 511-523. MR1612685 (99e:58169)

[11] S. Eilertsen, On weighted fractional integral inequalities, J. Funct. Anal. 185 (2001), 342-366. MR1853761 (2002h:26008)

[12] G. B. Folland and A. Sitaram, The uncertainty principle: a mathematical survey, J. Fourier Anal. Appl. 3 (1997), 207-238. MR1448337 (98f:42006)

[13] F. Gazzola, H.-Ch. Grunau and E. Mitidieri, Hardy inequalities with optimal constants and remainder terms, Trans. Amer. Math. Soc. 356 (2004), 2149-2168. MR2048513 (2005c:26031)

[14] G. H. Hardy, J. E. Littlewood and G. Pólya, Inequalities, Cambridge University Press, 1952. MR0046395 (13:727e)

[15] I. W. Herbst, Spectral theory of the operator $\left(p^{2}+m^{2}\right)^{1 / 2}-Z e^{2} / r$, Comm. Math. Phys. 53 (1977), 285-294. MR0436854 (55:9790)

[16] V. F. Kovalenko, M. A. Perelmuter and Ya. A. Semenov, Schrödinger operators with $L_{w}^{1 / 2}\left(\mathbb{R}^{l}\right)$ potentials, J. Math. Phys. 22 (1981), 1033-1044. MR622855 (83b:35121)

[17] E.H. Lieb, Sharp constants in the Hardy-Littlewood-Sobolev and related inequalities, Annals Math. 118 (1983), 349-374. MR717827 (86i:42010)

[18] H. R. Pitt, Theorems on Fourier series and power series, Duke Math. J. 3 (1937), 747-755. MR1546029

[19] S. Samko, Best constant in the weighted Hardy inequality: the spatial and spherical version, Fract. Calc. Anal. Appl. 8 (2005), 39-52. MR2179227 (2006m:26032)

[20] E. M. Stein, Singular integrals and differentiability properties of functions, Princeton University Press, 1970. MR0290095 (44:7280)

[21] E. M. Stein, Analytic continuation of group representations, Adv. Math. 4 (1970), 172-207. MR0263985 (41:8584)

[22] E. M. Stein, Harmonic analysis, Princeton University Press, 1995. MR1315539 (95i:42001)

[23] E. M. Stein and G. Weiss, Fractional integrals on n-dimensional Euclidean space, J. Math. Mech. 7 (1958), 503-514. MR0098285 (20:4746) 
[24] D. Yafaev, Sharp constants in the Hardy-Rellich inequalities, J. Funct. Anal. 168 (1999), 121-144. MR1717839 (2001e:26027)

[25] A. Zygmund, Trigonometric series, Cambridge University Press, 1959. MR0107776 (21:6498)

Department of Mathematics, The University of Texas at Austin, 1 University Station C1200, Austin, Texas 78712-0257

E-mail address: beckner@math.utexas.edu 